\title{
Chamfer Metrics, the Medial Axis and Mathematical Morphology
}

\author{
PETER F.M. NACKEN* \\ CWI, Kruislaan 413, 1098 SJ Amsterdam, The Netherlands
}

\begin{abstract}
This paper describes a number of efficient algorithms for morphological operations which use discs defined by chamfer distances as structuring elements. It presents an extension to previous work on extending metrics (such as the $p$-q-metrics). Theoretical results and algorithms are presented for $p-q-r$-metrics, which are not extending. These metrics can approximate the Euclidean metric close enough for most practical situations. The algorithms are based on an analysis of the structure of shortest paths in the $p-q-r$-metric and of the set of values this metric can assume. Efficient algorithms are presented for the medial axis and the opening transform. The opening transform algorithm is two orders of magnitude faster than a more straightforward algorithm.
\end{abstract}

\section{Introduction}

Mathematical morphology $[10,16]$ is an approach to image processing which is based on set theoretic notions such as inclusion and intersection. Recently, mathematical morphology has been extended to grey level images [16] and even complete lattices [6, 7, 15], but originally mathematical morphology dealt with binary images. Mathematical morphology models binary images as sets, by considering the foreground pixels as a subset of the image plane. The image is analyzed by inspecting the containment or intersection relations of the image with translates of some set $B$, the structuring element.

When mathematical morphology is applied to images defined on the real plane, one often uses discs as structuring elements. This is a suitable choice, because it makes morphological operators rotation invariant and because it allows for the interpretation of the results in terms of the natural metric for the image plane. Because discs are defined by a metric, it is possible to describe much of the theory of morphological operators based on circular structuring elements in terms

${ }^{*}$ This research was supported by the Foundation for Computer Science in The Netherlands (SION) with financial support from The Netherlands Organisation for Scientific Research (NWO). Part of it was performed while the author was a guest at the Technical University of Vienna in the framework of the Erasmus exchange program of the European Community. of metrics. In this approach, the notions of distance transformation and reconstruction occur in a natural way.

In practice, images are not defined on the real plane, but on (some subset of) the square grid $\mathbb{Z}^{2}$. On this grid, chamfer metrics [3] can be defined by setting the distance between pairs of neighboring points, and providing a rule for determining the distance between any pair of points from the distances between neighboring points. The definition of chamfer metrics is presented in the next section. The accuracy of a chamfer metric (compared with the Euclidean metric) can be improved by increasing the number of point pairs for which a prefixed distance is given. In typical cases, the distance of each point to eight other points is given, yielding the so-called $p$ - $q$-metrics, or the distance to 16 other points is given, yielding the so-called $p-q-r$ metrics.

Chamfer metrics can be used as accurate approximations to the Euclidean metric, at the same time allowing for efficient computation. In this paper, the metric approach to mathematical morphology will be combined with chamfer metrics in order to define the medial axis, opening transform and pattern spectrum associated with chamfer metrics. Efficient algorithms for these operations will be described.

The medial axis algorithm is different for $p-q$ metrics, such as the 3-4-metric or the 5-7-metric, and $p$ $q-r$-metrics such as the 5-7-11-metric. The author has 
described the algorithm for $p$-q-metrics in a previous paper [13]. This paper presents a medial axis algorithm for the $p-q-r$-metric. The algorithm for $p$ - $q$-metrics is presented in this paper for the sake of completeness, and in order to be able to point out the differences with the $p$ - $q-r$-metric.

The organization of the rest of the paper is as follows. The next section describes a number of notions from mathematical morphology and chamfer metrics and presents some properties of convex polygons. The tools presented in this section are used in Section 3 to derive the medial axis algorithm for the $p-q$-metric, and in Section 4 to present the medial axis algorithm for the $p-q-r$-metric. In Section 5, algorithms for the opening transform and pattern spectrum are derived, which use the medial axis algorithms derived in the previous sections. The last section sums up the conclusions of this paper.

\section{Chamfer Metrics and Mathematical Morphology}

In this section, we present the definitions discrete metrics and chamfer metrics and list some of their properties. For a proof of these properties, the reader is referred to a previous paper [13]. We also present some definitions and results on mathematical morphology, in particular the definitions of the medial axis, and some properties of convex polygons. We will write $D$ for the range $\{d(x, y) \mid x, y \in E\}$ of a metric $d$ on a set $E$.

Definition 2.1. A metric $d$ on a set $E$ is called a discrete metric if its range $D=\{d(x, y) \mid x, y \in E\}$ contains no limit points.

For a metric $d$, the closed sphere $\bar{B}(x, r)$ with center $x \in E$ and radius $r \in D$ is defined by

$$
\bar{B}(x, r)=\{y \in E \mid d(x, y) \leq r\} .
$$

One can associate two types of distance transforms with a given discrete metric.

Definition 2.2. Let $d$ be a discrete metric on $E$ and let $D$ be its range. The external distance transform $\rho_{X}^{\text {ext }}$ is the function $E \rightarrow D$ defined by

$$
\rho_{X}^{\mathrm{ext}}(x)=\min \left\{d(x, y) \mid y \in X^{C}\right\} .
$$

Definition 2.3. Let $d$ be a discrete metric on $E$ and let $D$ be its range. The internal distance transform $\rho_{X}^{\text {int }}$ is the function $X \rightarrow D$ defined by

$$
\rho_{X}^{\text {int }}(x)=\max \{r \in D \mid \bar{B}(x, r) \subseteq X\} .
$$

Because $D$ has no limit points, the equality

$$
\rho_{X}^{\mathrm{int}}(x)=\max \left\{r \in D \mid r<\rho_{X}^{\mathrm{ext}}(x)\right\}
$$

holds. This equality enables us to compute the internal distance transform from the external one when $D$ is known [13]. In the literature, the external distance transform is used most frequently. In this paper, the internal distance transform is most important, so from now on we will write $\rho$ for the internal distance transform.

A notion closely related to the distance transform is the (closed sphere) reconstruction.

Definition 2.4. Let $d$ be a discrete metric on $E$ and let $D$ be its range. Let $f$ be a function from a bounded subset $X$ of $E$ to $D$. The reconstruction $R(f)$ of $f$ is defined as the set

$$
R(f)=\bigcup_{x \in X} \bar{B}(x, f(x)) .
$$

This definition suggests that a reconstruction can be computed by a simple algorithm. A set is initialized to be empty, and the sphere $\bar{B}(x, f(x))$ is added to it for each $x \in X$. Note that for every bounded set $X$ the relation $X=R\left(\rho_{X}^{\text {int }}\right)$ holds.

We will now present the definition of the medial axis [16] and its relation to metrics. From the definition of the internal distance transform, it can easily be deduced that a sphere $\bar{B}(x, r)$ with center $x \in E$ and radius $r \in D$ is included in a set $X$ if and only if $r \leq \rho(x)$. The largest sphere with center $x$ which is contained in $X$ is therefore $\bar{B}(x, \rho(x))$.

Definition 2.5. Let $X$ be a bounded subset of $E$ and let $d$ be a discrete metric. The medial axis $M_{X}$ of $X$ is the locus of the centers of maximal spheres in $X$, i.e., $x \in M_{X}$ if and only if there is an $r \in D$ such that

$$
\bar{B}(x, r) \subseteq \bar{B}\left(x^{\prime}, r^{\prime}\right) \subseteq X \Rightarrow x^{\prime}=x, r^{\prime}=r .
$$

It can readily be seen that $x \in M_{X}$ if and only if there is no $y \in X$ such that $y \neq x$ and $\bar{B}(x, \rho(x)) \subseteq$ 
$\bar{B}(y, \rho(y))$. This property will be used throughout the next sections.

The homotopy of the medial axis of a set $X$ is in general different from the homotopy of the original set. A thin subset of $X$ which has the same homotopy as $X$ and lies in some sense "in the middle" of $X$ is often called a skeleton. The medial axis, skeletons and their relations have been investigated extensively in the past $[2,5,11-14,16]$.

A particular class of discrete metrics is formed by chamfer metrics [3]. Chamfer metrics were originally defined by Borgefors. In this paper, we restrict ourselves to chamfer metrics on the square grid $\mathbb{Z}^{2}$. The first step in the construction of a chamfer metric is the selection of a set of so-called prime vectors $\left\{v_{1}, \ldots, v_{k}\right\}$. Although not necessary from a theoretical point of view [13], it is common to choose a set of prime vectors which is invariant under the symmetry group $D_{4}$ of the square grid; $D_{4}$ consists of 4 rotations (including the identity) and 4 reflections in horizontal, vertical and diagonal lines. Common choices are to use the four vectors of the form $( \pm 1,0)$ or $(0, \pm 1)$, the eight vectors of the form $( \pm 1,0),(0, \pm 1)$ or $( \pm 1, \pm 1)$ or the sixteen vectors of the form $( \pm 1,0)$, $(0, \pm 1),( \pm 1, \pm 1),( \pm 1, \pm 2)$ or $( \pm 2, \pm 1)$. These sets of prime vectors correspond to the 4-neighborhood, the 8-neighborhood and the neighborhood containing the 8-neighbors and the pixels at a knight's mover from the center, respectively. The latter neighborhood will from now on be referred to as the 16neighborhood.

The next step is the assignment of a weight $l\left(v_{i}\right)$ to each prime vector. These weights are positive numbers; in this paper, we use integers only. The weights are chosen such that they are invariant under the symmetry group $D_{4}$ of the grid. Therefore, if eight prime vectors are chosen as mentioned above, the four vectors $( \pm 1,0)$ and $(0, \pm 1)$ must have the same weight $p$, and the four vectors $( \pm 1, \pm 1)$ must also have the same weight $q$. If sixteen prime vectors are chosen as mentioned above, the eight vectors $( \pm 1, \pm 2)$ and $( \pm 2, \pm 1)$ must have the same weight $r$. The resulting chamfer metrics will be called $p-q$-metrics and $p-q-r$-metrics, respectively.

The chamfer metric corresponding to a given set of prime vectors and their weights can now be defined as follows.

Definition 2.6. Let $\left\{v_{1}, \ldots, v_{k}\right\}$ be a set of prime vectors, invariant under $D_{4}$ and let $l\left(v_{i}\right)$ be their weights, also invariant under $D_{4}$. The chamfer metric $d$ on $\mathbb{Z}^{2}$ is defined by

$$
\begin{aligned}
& d(x, y) \\
& =\min \left\{\sum_{i} n_{i} l\left(v_{i}\right) \mid n_{i} \in \mathbb{N}, \sum_{i} n_{i} v_{i}=y-x\right\} .
\end{aligned}
$$

It is not hard to see that this definition is equivalent to the more common definition using paths, which one often encounters in the literature.

Suppose that no two prime vectors point in the same direction. Then the prime vectors can be ordered clockwise according to their direction. Two prime vectors will be called adjacent if they are adjacent in this ordering. An equivalent definition of adjacency is the following: two prime vectors $v_{1}$ and $v_{2}$ are adjacent if the only prime vectors in $\left\{\lambda_{1} v_{1}+\lambda_{2} v_{2} \mid \lambda_{1}, \lambda_{2} \in\right.$ $\left.\mathbb{R}, \lambda_{1}, \lambda_{2} \geq 0\right\}$ are $v_{1}$ and $v_{2}$. Let $\hat{v}_{i}=v_{i} / l\left(v_{i}\right)$ be the so called normalized prime vectors. These prime vectors will in general not be elements of the discrete $\operatorname{grid} \mathbb{Z}^{2}$. We will often use the following theorem [13]:

Theorem 2.7. Suppose that a set $\left\{v_{1}, \ldots, v_{k}\right\}$ with weights $l\left(v_{i}\right)$ satisfies the following properties:

(1) If $v_{1}=\left(x_{1}, y_{1}\right)$ and $v_{2}=\left(x_{2}, y_{2}\right)$ are adjacent prime vectors, then $x_{1} y_{2}-x_{2} y_{1}= \pm 1$.

(2) The normalized prime vectors $v_{i} / l\left(v_{i}\right)$ are the corners of a convex polygon.

Then each vector $x$ of the square grid can be written in a unique way in the form $n_{1} v_{1}+n_{2} v_{2}$, where $v_{1}$ and $v_{2}$ are adjacent prime vectors, $n_{1}$ and $n_{2}$ are nonnegative integers and $d(x, 0)=n_{1} l\left(v_{1}\right)+n_{2} l\left(v_{2}\right)$.

All chamfer metrics one usually comes across satisfy these conditions. If one finds a way of writing a vector $y-x$ as $n_{1} v_{1}+n_{2} v_{2}$ where $n_{1}, n_{2}, v_{1}$ and $v_{2}$ are as described above, it follows from the uniqueness of this expression that $d(x, y)=n_{1} l\left(v_{1}\right)+n_{2} l\left(v_{2}\right)$.

Note that, in general, not all integers occur in the range $D=\left\{d(x, 0) \mid x \in \mathbb{Z}^{2}\right\}$. It can be shown [13] that if the weights of adjacent prime vectors have greatest common divisor 1 , the range contains all but a finite number of natural numbers. The ranges of a $p$ - $q$-metric and a $p-q-r$-metric will sometimes be written as $D_{p-q}$ and $D_{p-q-r}$, respectively.

In Section 4, we will consider the extension of the $p-q-r$-metric to the continuous plane. It is defined as follows. 
Definition 2.8. Let $V$ be the set of prime vectors described above and let $l$ be the weight functions satisfying the conditions mentioned above. The $p-q-r$ chamfer metric on $\mathbb{R}^{2}$ is defined by

$$
\begin{aligned}
& d(x, y) \\
& \quad=\inf \left\{\sum_{i}\left|n_{i}\right| l\left(v_{i}\right) \mid n_{i} \in \mathbb{R}, \sum_{i} n_{i} v_{i}=y-x\right\}
\end{aligned}
$$

It is not difficult to see that the $p-q-r$-chamfer metric on $\mathbb{R}^{2}$ is indeed a metric, and that the "spheres" defined by this metric are polygons with 16 corners. From the proof of Lemma 2.7, it can be derived that the restriction of the $p$-q-r-metric on $\mathbb{R}^{2}$ to $\mathbb{Z}^{2}$ is the $p$-q-r-metric on $\mathbb{Z}^{2}$.

Spheres in the continuous plane are defined as fcllows.

Definition 2.9. For each $\rho \in \mathbb{R}, \rho \geq 0$ and $x \in \mathbb{R}^{2}$, the continuous sphere $S(x, \rho)$ is defined by

$$
S(x, \rho)=\left\{y \in \mathbb{R}^{2} \mid d(x, y) \leq \rho\right\} .
$$

Continuous and discrete spheres are related as follows. Suppose $x \in \mathbb{Z}^{2}$ and $\rho \in \mathbb{R}, \rho \geq 0$. Then

$$
S(x, \rho) \cap \mathbb{Z}^{2}=B\left(x, \rho^{-}\right),
$$

where $\rho^{-}$is the number $\max \{\tau \in D \mid \tau \leq \rho\}$. This implies that, for $x \in \mathbb{Z}^{2}$ and $\rho \in D$,

$$
B(x, \rho)=S(x, \rho) \cap \mathbb{Z}^{2} .
$$

For continuous spheres, the equivalence $S(x, \rho) \subseteq$ $S(y, \tau) \Leftrightarrow \tau \geq \rho+d(x, y)$ holds. As noted before [13], this relation is not true for discrete spheres.

Definition 2.10. The relation $\sqsubseteq$ between subsets $A$ and $B$ of $\mathbb{R}^{2}$ is defined by

$$
A \subseteq B \text { iff } A \cap \mathbb{Z}^{2} \subseteq B \cap \mathbb{Z}^{2} .
$$

Obviously, $A \subseteq B$ implies $A \sqsubseteq B$, but the reverse is in general not true.

The following lemmas describe the behavior of convex sets under scalings. They will be used in Section 4. The scaling $M(c, \lambda)(X)$ of a subset $X$ of $\mathbb{R}^{2}$ with center $c \in \mathbb{R}^{2}$ and magnification factor $\lambda \in \mathbb{R}$ is defined as the set $\{\lambda(x-c)+c \mid x \in X\}$.

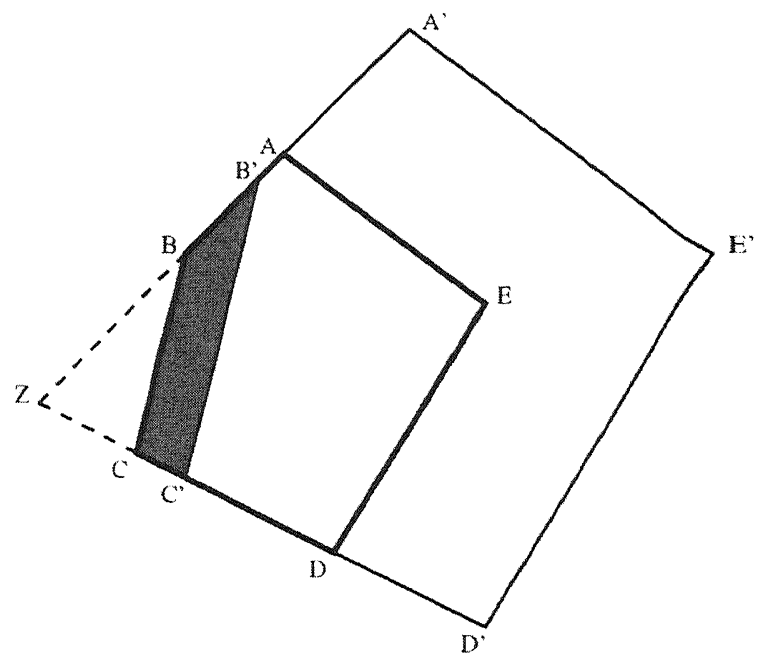

Figure 1. Illustration of Lemma 2.12. The only part of the small polygon $A B C D E$ which is not contained in the magnified polygon $A^{\prime} B^{\prime} C^{\prime} D^{\prime} E^{\prime}$ is the shaded area.

Lemma 2.11. Let $X$ be a convex subset of $\mathbb{R}^{2}$ and let $x \in X, \lambda>1$. Then $X \subseteq M(x, \lambda)(X)$.

Lemma 2.12. Let $P$ be a convex polygon. Suppose that the carriers of two of its sides $A B$ and $C D$ intersect in a point $Z$, and that $B$ is closer to $Z$ than $A$ and $C$ is closer to $Z$ than $D$. Suppose $\lambda$ is a scaling factor such that $B^{\prime}=\lambda(B-Z)+Z$ lies between $B$ and $A$ and $C^{\prime}=\lambda(C-Z)+Z$ lies between $C$ and $D$. Then the only part of $P$ which is not contained in $M(Z, \lambda)(P)$ is the polygon bounded by the boundaries of the polygons between $B$ and $C$ and between $B^{\prime}$ and $C^{\prime}$, and the segments $B B^{\prime}$ and $C C^{\prime}$.

This lemma is illustrated in Fig. 1. It can be proven by considering, for all lines $l$ through $Z$, the intersections of $l$ with $P$ and $M(Z, \lambda)(P)$. These intersections are either both equal to $\emptyset$ or partially overlapping segments of $l$.

\section{The Medial Axis for the $p-q$-Metric}

In this section, we present the medial axis algorithm for $p$-q-metrics. This algorithm uses a no-upstream condition for the internal distance transform which is analogous to the no-upstream condition which holds for the medial axis in $\mathbb{R}^{2}$ [11]. Recall that, if $X$ is some open subset of $\mathbb{R}^{2}$, its distance transform $\rho$ is the function from $\mathbb{R}^{2}$ to $\mathbb{R}$ defined by $\rho(x)=\inf \{d(x, y) \mid$ $\left.y \in X^{C}\right\}$. A point $x \in X$ is a medial axis point if and only if there is no $y \neq x$ such that $\rho(y)=\rho(x)+$ $d(x, y)$. 
The algorithm presented in this section for $p$ $q$-metrics uses the fact that these metrics are extending.

Definition 3.1. Let $d$ be a metric on a set $E$ with range $D$. A value $r \in D$ is called an extending value if for each $x, y \in E$ there is a $z \in E$ such that $d(x, y)+$ $d(y, z)=d(x, z)$ and $d(y, z)=r$.

Definition 3.2. A metric $d$ on a set $E$ with range $D$ is called extending if each $r \in D$ is an extending value.

The Euclidean metric is extending. Given $x, y$ and $r$, the point $z$ as described in the definition can be constructed using simple geometry: it is one of the intersections of the line $x y$ with the circle with center $y$ and radius $r$. Of the two intersection points, the one must be chosen for which $y$ lies between $x$ and $z$.

All $p$-q-metrics are extending as well. This can be shown by constructing a point $z$, as we did in the previous paragraph for the Euclidean metric. Let $x$ and $y$ be two points in $\mathbb{Z}^{2}$ and let $r \in D_{p-q}$. The vector $y-x$ can be written in the form $n_{1} v_{1}+n_{2} v_{2}$, where $v_{1}$ and $v_{2}$ are adjacent prime vectors and $n_{1}$ and $n_{2}$ are non-negative integers. Without loss of generality, it can be assumed that $l\left(v_{1}\right)=p$ and $l\left(v_{2}\right)=q$. As $r \in D_{p-q}, r$ can be written as $m_{1} p+m_{2} q$, where $m_{1}$ and $m_{2}$ are nonnegative integers. We can take $z=y+m_{1} v_{1}+m_{2} v_{2}$. Then $z-y=m_{1} v_{1}+m_{2} v_{2}$, so $d(y, z)=m_{1} p+m_{2} q=r$. Moreover, $z-x=\left(n_{1}+m_{1}\right) v_{1}+\left(n_{2}+m_{2}\right) v_{2}$, so $d(x, z)=\left(m_{1}+n_{1}\right) p+\left(m_{2}+n_{2}\right) q=d(x, y)+$ $d(y, z)$.

The following theorem presents the no-upstream condition for medial axis points for extending metrics.

Theorem 3.3. Let $d$ be a metric on $\mathbb{Z}^{2}$. Let $X$ be a bounded subset of $\mathbb{Z}^{2}$. A point $x \in X$ for which $\rho(x)$ is an extending value is the center of a maximal sphere if and only if there is no $y \neq x$ such that $\rho(y) \geq$ $\rho(x)+d(x, y)$ (" $x$ has no upstream").

Proof: 'Only if': suppose that there exists an $y \neq x$ such that $\rho(y) \geq \rho(x)+d(x, y)$. In that case every $z \in \bar{B}(x, \rho(x))$ satisfies

$d(z, y) \leq d(z, x)+d(x, y) \leq \rho(x)+d(x, y) \leq \rho(y)$.

Therefore $\bar{B}(y, \rho(x))$ is a sphere containing $\bar{B}(x, \rho(x))$ and contained in $X$. Therefore, $x$ is not the center of a maximal sphere.
'If': suppose $x$ is not the center of a maximal sphere. Then the sphere with center $x$ and radius $\rho(x)$ must be contained in a closed sphere with center $y \neq x$ and radius $\rho(y)$. Let $z$ be a point such that $d(y, x)+d(x, z)=d(y, z)$ and $d(x, z)=\rho(x)$. Such a points exists because $\rho(x)$ is an extending value. From $z \in \bar{B}(x, \rho(x)) \subseteq \bar{B}(y, \rho(y))$ it follows that $d(z, y) \leq \rho(y)$. From these relations it can be deduced that

$$
\begin{aligned}
\rho(y)-\rho(x) & \geq d(z, y)-\rho(x) \\
& =d(z, y)-d(x, z)=d(x, y) .
\end{aligned}
$$

Corollary 3.4. Let $d$ be an extending metric on $\mathbb{Z}^{2}$ and $X$ a bounded subset. Then $x \in X$ is a medial axis point if and only if there is no $y \in X$ such that $\rho(y) \geq$ $\rho(x)+d(x, y)$.

This theorem provides a characterization of the medial axis points, but it cannot be used for the construction of an efficient algorithm: in order to determine whether $x$ is a medial axis point of $X$, all points $y$ must be inspected. In the case of the $p-q$-metric, the search can be limited to the neighbors of $x$.

Theorem 3.5. Let $X$ be a bounded subset of $\mathbb{Z}^{2}$, provided with a chamfer metric whose set of prime vectors is $V$ and let $x \in X$. If there is a point $y$ such that $\rho(y) \geq \rho(x)+d(x, y)$, then there is also an $y^{\prime} \in \mathbb{Z}^{2}$ satisfying $y^{\prime}-x \in V$ and $\rho(x)+d\left(x, y^{\prime}\right)$.

Proof: Let $x$ and $y$ be points as described in the theorem. Then

$$
\bar{B}(x, \rho(x)) \subseteq \bar{B}(y, \rho(y)) \subseteq X .
$$

There is a pair of adjacent prime vectors $v_{1}$ and $v_{2}$ such that $x-y=n_{1} v_{1}+n_{2} v_{2}$ and $n_{1}$ and $n_{2}$ are nonnegative. Without loss of generality, it can be assumed that $n_{1}>0$. We can now take $y^{\prime}=x-v_{1}$. This implies $d(x, y)=d\left(x, y^{\prime}\right)+d\left(y^{\prime}, y\right)$ and $y^{\prime}-x \in V$. It is now sufficient to prove that

$$
\bar{B}(x, \rho(x)) \subseteq \bar{B}\left(y^{\prime}, \rho(x)+d\left(x, y^{\prime}\right)\right) \subseteq \bar{B}(y, \rho(y)) .
$$

The first inclusion follows from

$$
\begin{aligned}
d(x, p) \leq \rho(x) & \Rightarrow d\left(y^{\prime}, p\right) \leq d\left(y^{\prime}, x\right)+d(x, p) \\
& \leq d\left(y^{\prime}, x\right)+\rho(x) .
\end{aligned}
$$


The second inclusion follows from:

$$
\begin{aligned}
d\left(y^{\prime}, p\right) & \leq \rho(x)+d\left(x, y^{\prime}\right) \Rightarrow d(y, p) \\
& \leq d\left(y, y^{\prime}\right)+d\left(y^{\prime}, p\right) \\
& \leq d\left(x, y^{\prime}\right)+d\left(y^{\prime}, y\right)+\rho(x) \\
& =d(x, y)+\rho(x) \leq \rho(y) .
\end{aligned}
$$

Corollary 3.6. Let $X$ be a bounded subset of $\mathbb{Z}^{2}$, provided with the $p$-q-metric. Then $x \in X$ is a medial axis point if and only if there is no 8-neighbor $y$ of $x$ such that $\rho(y) \geq \rho(x)+d(x, y)$.

This theorem suggests the following algorithm for the computation of the medial axis in the $p-q$-metric.

Algorithm 3.7. The computation of the medial axis of a bounded subset $X$ of $\mathbb{Z}^{2}$ with respect to the $p-q$ metric.

(1) Compute the external distance transform of $X$.

(2) Compute the internal distance transform $\rho$ of $X$ from the external one.

(3) Mark all points $x$ having no 8-neighbor $y$ with $\rho(y) \geq \rho(x)+d(x, y)$.

This algorithm requires four image scans, and local computation only. It is possible to perform steps (2) and (3) in a single scan, but this makes the local operation to be performed in this step much more complicated, so this is not a good approach.

\section{The Medial Axis for the $p-q-r$-Metric}

A $p$ - $q$-r-chamfer metric can approximate the Euclidean metric more accurately than any $p-q$-metric [18]. The relative error of the 5-7-11-metric with respect to the Euclidean metric is $1.79 \%$, while the relative error of any $p$-q-metric is at least $3.96 \%$. This implies that the error made when measuring an object of diameter 50 is in the order of a single pixel when the 5-7-11-metric is used. Therefore, the accuracy of the 5-7-11-metric is sufficient in most practical situations.

On the other hand, the algorithms for the $p-q-r$ metric are more complicated than those for the $p-q$ metric. The medial axis algorithm described in the previous section cannot be used, because the $p-q-r$ metric is not extending. This can be seen from the example in Fig. 2 for the 5-7-11-metric. The small sphere of radius 5 is contained in the larger one of

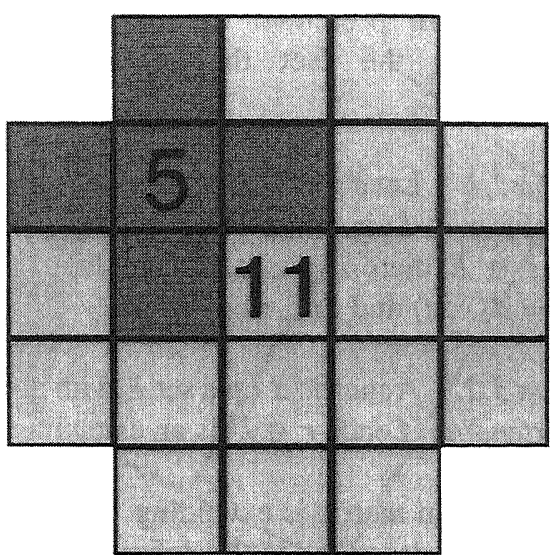

Figure 2. An example showing that the no-upstream criterion for the medial axis cannot be applied to the 5-7-11 metric.

radius 11 , but the distance of their centers is 7 , while the difference of their radii is only 6 . This section presents an algorithm which is suitable for the $p-q-r$-metric.

For the $p-q-r$-metric, there are three types of prime vectors: those of the form $( \pm 1,0)$ or $(0, \pm 1)$ with weight $p$, those of the form $( \pm 1, \pm 1)$ with weight $q$, and those of the form $( \pm 1, \pm 2)$ or $( \pm 2, \pm 1)$ with weight $r$. We will say that these vectors are of type $p$, type $q$ or type $r$, respectively. Each vector of type $r$ is adjacent to a vector of type $p$ and a vector of type $q$, but no pair of vectors of type $p$ and type $q$ are adjacent (see Fig. 3). As a consequence, each shortest path between two points contains either vectors of type $p$ and type $r$, vectors of type $q$ and type $r$ or vectors of a single type. The range $D_{p-q-r}$ of the $p-q-r$-metric consists of the points which can be written as $p x+r y$ or $q x+r y$, for nonnegative integers $x$ and $y$. Note that integers larger that $q r$ can be written in both forms (see [13] for a discussion of the structure of $D$ ).

Like in the previous section, our aim is to determine for each point $x$ whether there is a point $y \neq x$ such that $\bar{B}(x, \rho(x)) \subseteq \bar{B}(y, \rho(y))$. We will not try to find a criterion which decides whether this relation holds for two arbitrary points $x$ and $y$. Rather, we will show that if, for a given $x$, such a point $y$ exists, there is also a 16neighbor $y^{\prime}$ of $x$ such that $\bar{B}(x, \rho(x)) \subseteq \bar{B}\left(y^{\prime}, \rho\left(y^{\prime}\right)\right)$. It is then sufficient to check for each 16-neighbor $y$ of $x$ whether $\bar{B}(x, \rho(x)) \subseteq \bar{B}(y, \rho(y))$. If there is no such point, then $x$ is a medial axis point, otherwise it is not.

Theorem 4.1. Let $X$ be a bounded subset of $\mathbb{Z}^{2}$. A point $x \in X$ is a medial axis point if and only if 
there is no 16-neighbor $y$ of $x$ such that $B(x, \rho(x)) \subset$ $B(y, \rho(y))$.

Note that, if $\rho(x) \in p \mathbb{N}+r \mathbb{N}$ and $\rho(x) \in q \mathbb{N}+r \mathbb{N}$, $\rho(x)$ is an extending value and Theorem 3.3 can be used. It can then be argued, like in the proof of Theorem 3.5, that $x$ is a medial axis point if there is no 16-neighbor $y$ of $x$ such that $\rho(y) \geq \rho(x)+d(x, y)$. Yet, for the $p$-q-r-metric, there are values of $\rho(x)$ which are in $p \mathbb{N}+r \mathbb{N}$ but not in $q \mathbb{N}+r \mathbb{N}$, or vice versa.

The proof of Theorem 4.1 consists of two steps. First, we show (Lemma 4.3) that it is sufficient to inspect a star-shaped region, whose shape depends on $\rho(x)$. From this result, it will be derived (Lemma 4.4) that it is sufficient to inspect a 16 point neighborhood.

In order to simplify the discussion, we will suppose that $x$ is the origin. We divide $\mathbb{Z}^{2}$ in eight octants, as shown in Fig. 3. The octants will be called the $(2,1)$ octant, the $(1,2)$-octant, the $(-1,2)$-octant, etc., after the prime vector of type $r$ they contain. Note that the symmetry group $D_{4}$, which consists of four rotations (including the identity) and four reflections in horizontal, vertical and diagonal lines, maps a point in one octant to a point in each of the other octants. Therefore we can assume without loss of generality that $y$ lies in the $(-2,-1)$-octant.

Suppose that $z$ is a point in the $(2,1)$-octant. Let $z_{1}=z, z_{2}, \ldots, z_{8}$ be the images under $D_{4}$ of the point $z$. It can easily be seen that $d\left(y, z_{i}\right) \leq d(y, z)$ for all $i$. Consequently, the sphere $\bar{B}(0, \rho(0))$ is completely

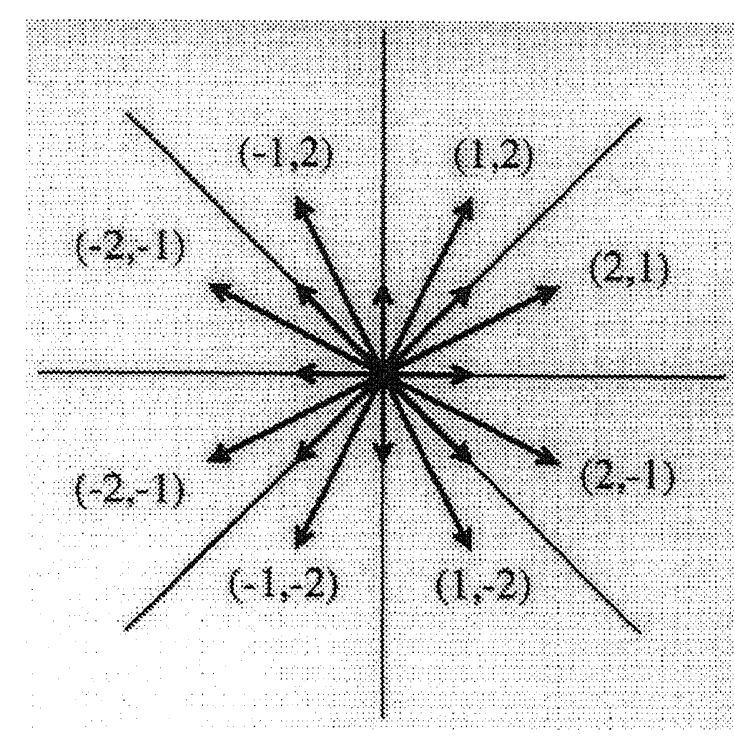

Figure 3. Horizontal, vertical and diagonal vectors partition the plane in eight parts, which can be labeled according the vector of type $q$ they contain.

contained in $\bar{B}(y, \rho(y))$ if the intersection of the sphere centered at 0 with the $(2,1)$-octant is completely contained in the sphere centered at $y$.

As a consequence of the two previous paragraphs, we may restrict ourselves to the situation depicted in Fig. 4. Let $l, m$ and $n$ be the lines through the origin in the directions $(1,1),(2,1)$ and $(1,0)$, respectively. Let $P$ and $Q$ be the points

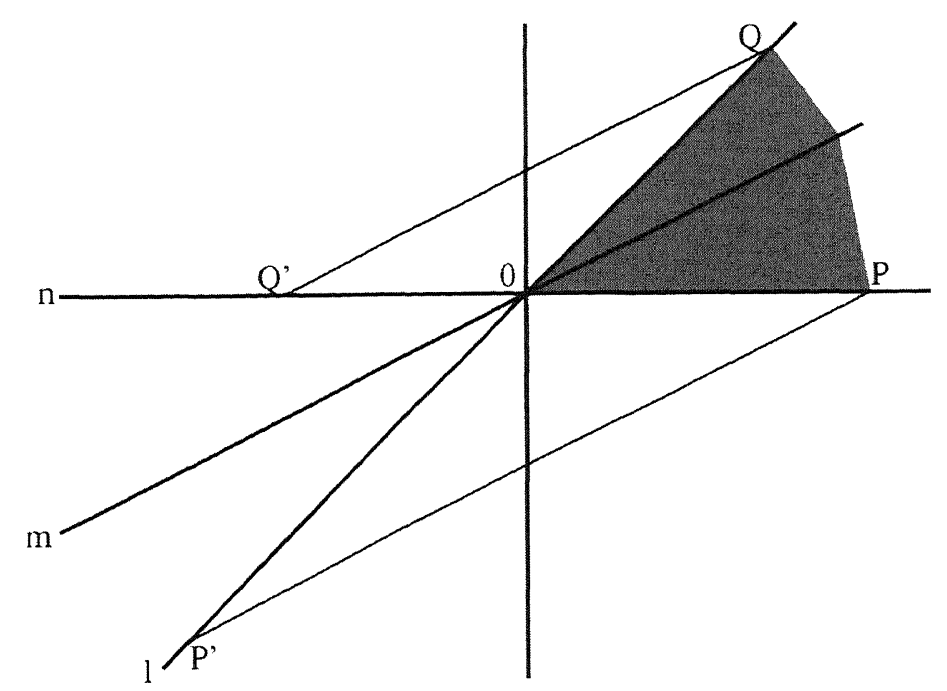

Figure 4. The geometry of the situation under consideration. See the text for an explanation. 
$(\lfloor\rho(0) / p\rfloor, 0)$ and $(\lfloor\rho(0) / q\rfloor,\lfloor\rho(0) / q\rfloor)$ and le $=$ $(-\lfloor\rho(0) / p\rfloor,-\lfloor\rho(0) / p\rfloor)$ and $Q^{\prime}=(-\lfloor\rho(0) / q\rfloor, 0)$ be their projections on the lines $l$ and $n$ in the $(2,1)$ direction. The shaded region represents the intersection of $\bar{B}(0, \rho(0))$ with the $(2,1)$-octant. This region will be referred to as the opposite part of the sphere. It lies above the line $P P^{\prime}$ and below the line $Q Q^{\prime}$.

Let $y$ be a point in the $(-2,-1)$-octant such that $\bar{B}(0, \rho(0)) \subseteq \bar{B}(y, \rho(y))$. We are now ready to construct a point $y^{\prime}$ near 0 such that $\bar{B}(0, \rho(0)) \subseteq$ $\bar{B}\left(y^{\prime}, \rho\left(y^{\prime}\right)\right)$ as well.

Lemma 4.2. Let $y$ be a point in the $(-2,-1)$ octant such that $\bar{B}(0, \rho(0)) \subseteq \bar{B}(y, \rho(y))$. Then $\bar{B}(0, \rho(0)) \subseteq \bar{B}\left(y^{\prime}, \rho\left(y^{\prime}\right)\right)$ if $y^{\prime}$ satisfies the following conditions:

(1) $y^{\prime}$ lies in the $(-2,-1)$-octant.

(2) $d(y, z)=d\left(y, y^{\prime}\right)+d\left(y^{\prime}, z\right)$ for every $z$ in the opposite part of the sphere.

Proof: It is sufficient to show that

$$
\bar{B}(0, \rho(0)) \subseteq \bar{B}\left(y^{\prime}, \rho(y)-d\left(y, y^{\prime}\right)\right) \subseteq \bar{B}(y, \rho(y)) .
$$

As $y^{\prime}$ lies in the $(-2,-1)$-octant, the first inclusion can be proved by showing that $d\left(y^{\prime}, z\right) \leq \rho(y)-d\left(y, y^{\prime}\right)$ for each point $z$ in the opposite part, i.e., for each $z$ in the $(2,1)$-octant with $d(z, 0) \leq \rho(0)$. But according to (2), we have for such points $z: d\left(y^{\prime}, z\right)=d(y, z)-$ $d\left(y, y^{\prime}\right)$. Because $\bar{B}(0, \rho(0)) \subseteq \bar{B}(y, \rho(y))$ we know $d(y, z) \leq \rho(y)$, so $d\left(y^{\prime}, z\right) \leq \rho(y)-d\left(y, y^{\prime}\right)$.

The second inclusion holds because $d\left(z, y^{\prime}\right) \leq$ $\rho(y)-d\left(y, y^{\prime}\right)$ implies $d(z, y) \leq d\left(z, y^{\prime}\right)+d\left(y^{\prime}, y\right) \leq$ $\rho(y)$.

Lemma 4.3. Let $X$ be a bounded subset of $\mathbb{Z}^{2}$. A point $x \in X$ is a medial axis point if there is no point $y$ with $B(x, \rho(x)) \subseteq B(y, \rho(y))$ and one of the following holds:

(1) $y-x=(a, 0)$ or $(0, a)$, with $|a| \leq \rho(x) / q$.

(2) $y-x=(a, a)$ or $(a,-a)$, with $|a| \leq \rho(x) / p$.

(3) $y-x=( \pm 1, \pm 2)$ or $( \pm 2, \pm 1)$.

Proof: Without loss of generality, we can consider the situation of Fig. 4. We will construct a point $y^{\prime}$ which satisfies the conditions mentioned in Lemma 4.2. The point $y$ lies in one of five regions (see Fig. 5).

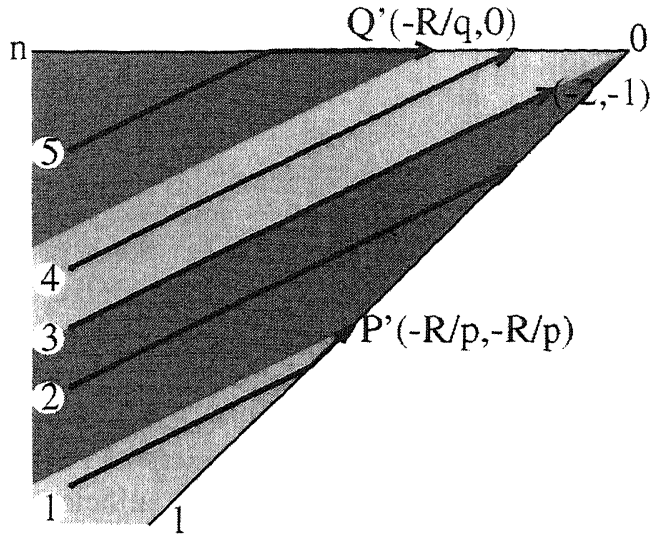

Figure 5. The construction of the points $y^{\prime}$ from the points $y$ on the left. The points $y^{\prime}$ are found by moving in the $(2,1)$-direction until the line $l$ or $n$ is hit. If the line is hit to the left of $P^{\prime}$ or $Q^{\prime}$, move rightward along the line to one of these points. Regions 1 , 2,4 and 5 are the shaded areas; region 3 is the half line ending at $(-2,-1)$.

Region 1. $y=-\alpha(2,1)-\beta(1,1)$ with $\alpha \geq 0, \beta \geq$ $\lfloor\rho(0) / p\rfloor$. Then we take $y^{\prime}=P^{\prime}$. Clearly, $P^{\prime}$ lies in the $(-2,-1)$-octant. We have $P^{\prime}-y=\alpha(2,1)+(\beta-$ $\lfloor\rho(0) / p\rfloor)(1,1)$, a nonnegative linear combination of $(2,1)$ and $(1,1)$. Because all points in the opposite part of the sphere lie above the line $P P^{\prime}$ and below $l$, it is possible for each point $z$ in the opposite part to write $z-P^{\prime}$ as a nonnegative linear combination of $(1,1)$ and $(2,1)$ as well. This implies $d\left(y, P^{\prime}\right)+d\left(P^{\prime}, z\right)=$ $d(y, z)$.

Region 2. Suppose $y=-\alpha(2,1)-\beta(1,1)$ with $\alpha$ geq $0,0<\beta<\lfloor\rho(0) / p\rfloor$. Then we take $y^{\prime}=-$ $\beta(1,1)$. Clearly, this $y^{\prime}$ lies in the $(-2,-1)$-octant. We have $y^{\prime}-y=\alpha(2,1)$. Because the opposite part lies below the line $l$ and above the $x$-axis, it is possible for all points $z$ in the opposite part to write $z-y^{\prime}$ as a nonnegative linear combination of either $(1,1)$ and $(2,1)$ or $(2,1)$ and $(1,0)$. In both cases, $d\left(y, y^{\prime}\right)+d\left(y^{\prime}, z\right)=d(y, z)$.

Region 3. Suppose $y=-\alpha(2,1)$ with $\alpha>0$. Then we take $y^{\prime}=(-2,-1)$. Clearly $y^{\prime}$ lies in the $(-2,-1)$ octant. We have $y^{\prime}-y=-(\alpha-1)(2,1)$. As the opposite part lies below the line $l$ and above $x$-axis, it is possible write for each $z$ in the opposite part to write $z-y^{\prime}$ as a nonnegative linear combination of either $(1,1)$ and $(2,1)$ or $(2,1)$ and $(0,1)$. In both cases $d\left(y, y^{\prime}\right)+d\left(y^{\prime}, z\right)=d(y, z)$. 


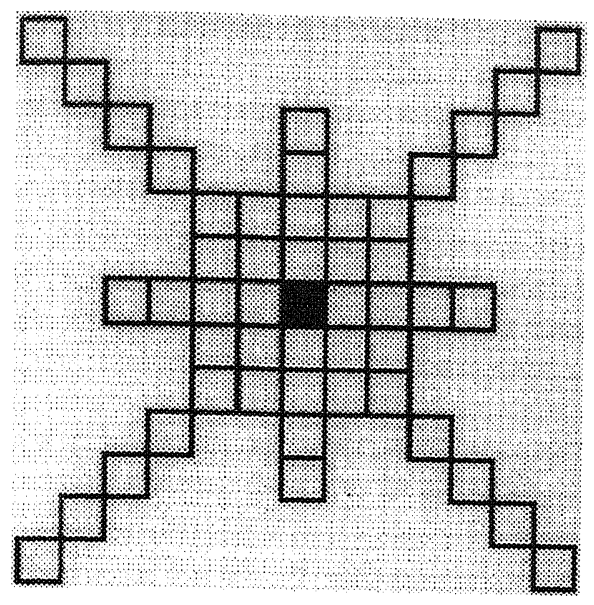

Figure 6. The neighborhood which must be investigated for a point with a distance transform value of 31 .

Region 4. Suppose $y=-\alpha(2,1)-\beta(1,0)$ with $\alpha \geq$ $0,0<\beta<\lfloor\rho(0) / q\rfloor$. Take $y^{\prime}=-\beta(1,0)$, as we did for $y$ in region 2 .

Region 5. Suppose $y=-\alpha(2,1)-\beta(1,0)$ with $\alpha \geq$ $0, \beta \geq\lfloor\rho(0) / q\rfloor$. Take $y^{\prime}=Q^{\prime}$, as we did for $y$ in region 1 .

We have now arrived at a local neighborhood of the origin which must be inspected in order to determine if the origin is a medial axis point. This environment contains $\lfloor\rho(0) / q\rfloor$ points in each of the four horizontal or vertical directions, $\lfloor\rho(0) / p\rfloor$ points in each of the four diagonal directions and eight points at a knights jump from the origin. Of course, similar environments for other points can be found by translation. Note that the size of the environment depends on the value of $\rho(x)$. Figure 6 shows the environment corresponding to $\rho(0)=31$ for the 5-7-11-metric. The environment contains horizontal and vertical branches of length $\left\lfloor\frac{31}{7}\right\rfloor=4$, diagonal branches of length $\left\lfloor\frac{31}{5}\right\rfloor=$ 6 and eight points at a knights move from the center.

We will now show that it is possible to restrict the neighborhood to sixteen points. Therefore, we must consider the case where $y$ is a point on an axis or on a diagonal, but not a neighbor of $x$.

Lemma 4.4. Let $\rho, \tau \in D, a \in \mathbb{N}$ with $a>1$.

(1) If $B((0,0), \rho) \subseteq B((-a, 0), \tau)$, then there is an $\rho^{\prime} \in D$ such that

$$
B((0,0), \rho) \subseteq B\left((-1,0), \rho^{\prime}\right) \subseteq B((-a, 0), \tau)
$$

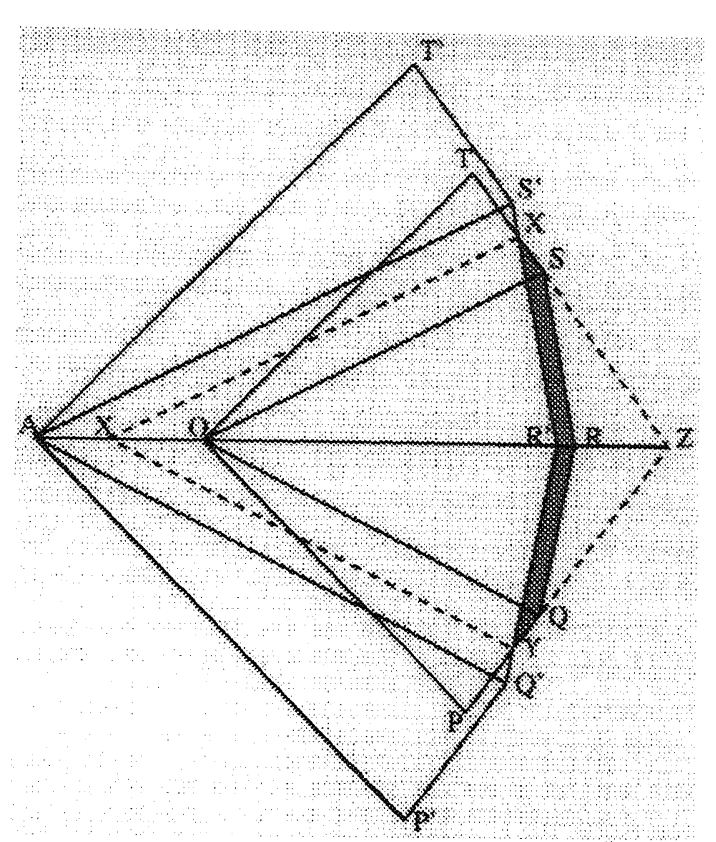

Figure 7. The relative positions of two spheres. See the text for an explanation.

(2) If $B((0,0), \rho) \subseteq B((-a,-a)$, $\tau)$, then there is an $\rho^{\prime} \in D$ such that

$$
B((0,0), \rho) \subseteq B\left((-1,-1), \rho^{\prime}\right) \subseteq B((-a,-a), \tau)
$$

Proof: We will prove only the first part of the lemma, as the second part can be proved in a similar way. It can be assumed without loss of generality that $\tau$ is the smallest value in $D$ for which $B((0,0), \rho) \subseteq$ $B((-a, 0), \tau)$ holds. The theorem is shown by considering continuous spheres in stead of discrete ones. We will prove that there is an $\rho^{\prime} \in \mathbb{R}$ such that

$S((0,0), \rho) \sqsubseteq S\left((-1,0), \rho^{\prime}\right) \sqsubseteq S((-a, 0), \tau)$.

The lemma follows immediately from this relation by applying (2.2) and Definition 2.10.

Consider the octagons $S((0,0), \rho)$ and $S((-a, 0)$, $\tau)$. Parts of these polygons are depicted in Fig. 7. The center of the small sphere is the origin $O$. Some of its corners, $P, Q, R, S$ and $T$, are marked. The center of the larger sphere is $A=(-a, 0)$; some of its corners, $P^{\prime}, Q^{\prime}, R^{\prime}, S^{\prime}$ and $T^{\prime}$, are marked.

There must be a grid point on the segment $R S$ or on the segment $S T$. If $S^{\prime}$ lies below the line $S T$, both the segments $R S$ and $S T$ lie outside the larger sphere, 
and so does at least one grid point. This would violate the inclusion relation. Therefore, $S^{\prime}$ lies above the line $S T$.

If $S^{\prime}$ lies above the line $R S$, the smaller sphere would be included in the interior of the larger one, and it would be possible to find a value $s^{\prime}<s$ for which $S((0,0), r) \subseteq S\left((0,-a), s^{\prime}\right)$ holds. This would imply $B((0,0), r) \subseteq B\left((0,-a),{s^{\prime}}^{-}\right)$, violating the assumption that $s$ is the smallest value for which this inclusion holds. Therefore, $S^{\prime}$ lies below the line $R S$.

The boundaries of the spheres intersect in the points $X$ and $Y$. The projection of these points in the $(2,1)-$ respectively the $(2,-1)$-directions is the point $B$. Note that two degenerate cases can occur. The points $R$ and $R^{\prime}$ can coincide, or the point $S^{\prime}$ can lie on the segment $S T$. In these situations, $X$ will be chosen to be $S$ or $S^{\prime}$, respectively.

Note that the relation $S((0,0), \rho) \subseteq S((-a, 0), \tau)$ holds only if $R$ and $R^{\prime}$ coincide. On the other hand, since $S((0,0), \rho) \sqsubseteq S((-a, 0), \tau)$, the shaded area $Y Q R S X R^{\prime}$ does not contain any points from $\mathbb{Z}^{2}$.

The proof is as follows. We construct a family of spheres $S((-t, 0), r(t))$ such that $r(0)=\rho, r(a)=\tau$ and

$$
S((-t, 0), r(t)) \sqsubseteq S\left(\left(-t^{\prime}, 0\right), r\left(t^{\prime}\right)\right)
$$

if $t \leq t^{\prime}$. This implies

$$
S((0,0), r(0)) \sqsubseteq S((-t, 0), r(t)) \sqsubseteq S((-a, 0), s)
$$

for any $t \in[0, a]$. Then (4.1) follows directly by taking $t=1$ in (4.3).

The family $S((-t, 0), r(t))$ is found by transforming $S((0,0), \rho)$ gradually into $S((-a, 0), \tau)$. Intuitively, the family can be described as the sequence of spheres which is obtained by gradually shifting and enlarging $S((0,0), \rho)$ in such a way that $S$ moves along the segment $S X$ to $X$, and then along the segment $X S^{\prime}$ to $S^{\prime}$, while the center of the sphere moves from $(0,0)$ to $(-a, 0)$.

The family consists of two parts. The first part is obtained by applying the transformation $M(Z, \lambda)$ to $S((0,0), r)$ with $\lambda$ ranging from 1 to $\left|R^{\prime} X\right| /|R S|$. This yields the spheres $S((-t, 0), r(t))$ with $t$ between 0 and $b$.

The second part of the family is obtained by applying the transformation $M\left(R^{\prime}, \lambda\right)$ to the sphere $S((-b, 0), r(b))$, with $\lambda$ ranging from 1 to
$\left|R^{\prime} S^{\prime}\right| /\left|R^{\prime} X\right|$. This yields the spheres $S((-t, 0), r(t))$ with $t$ between $b$ and $a$.

It remains to be shown that the family thus obtained satisfies (4.2). For the first part of the family, this follows from Lemma 2.12, combined with the fact that the shaded area in Fig. 7 contains no grid points. For the second part, increasingness with respect to $\subseteq$ follows immediately from Lemma 2.11. Therefore, increasingness with respect to $\sqsubseteq$ certainly holds.

Now Theorem 4.1 follows directly from Lemma 4.3 and Lemma 4.4.

Theorem 4.1 implies that it is sufficient to consider 16-point neighborhoods in the detection of medial axis point. We will now describe the operations which are to be performed in such a neighborhood, and the resulting algorithm will be presented.

Let $x \in X$ be a point of a set $X \subset \mathbb{Z}^{2}$. In order to determine whether $x$ is a medial axis point, it must be checked for each neighbor $y$ of $X$ in a 16-point environment, whether

$$
B(x, \rho(x)) \subseteq B(y, \rho(y))
$$

holds. There are three cases to be discerned: $y$ is a direct (4-connected) neighbor of $x, y$ is an indirect (8connected but not 4-connected) neighbor of $x$, or $y$ is at a knights move from $x$.

In the last case, $d(y, p)=d(y, x)+d(x, p)=$ $r+d(x, p)$ for all $p$ in the $(2,1)$-octant. Therefore, $B(x, \rho(x)) \subseteq B(y, \rho(y))$ if and only if $\rho(y) \geq$ $\max \{d(y, p) \mid p \in B(0, \rho(0,0))\}=r+\rho(0)$.

Now suppose that $y$ is a direct neighbor of $x$. It can be assumed without loss of generality that $x=(0,0)$ and $y=(-1,0)$. Then $(4.4)$ is true if and only if $\rho(-1,0) \geq \rho(0,0)+\Delta \rho$, where $\Delta \rho$ is given by

$$
\begin{gathered}
\Delta \rho=\max \{d((-1,0), z)-\rho(0) \mid z \\
\in B((0,0), \rho(0,0))\}
\end{gathered}
$$

Consider again Fig. 7, and suppose that $A$ is the point $(-1,0)$.

The point $z$ which maximizes $d((-1,0), z)$ in (4.5) is a grid point on the boundary of the larger sphere which is also included in the smaller sphere. If $S^{\prime}$ lies strictly above the line $S T, z$ lies on the segment $R^{\prime} X$, but if $S^{\prime}$ lies on the segment $S T$, then $z$ can also lie on the segment $X T$.

In the first case, $d((0,0), z)=\max \{s \in p \mathbb{N}+r \mathbb{N} \mid$ $s \leq \rho(0,0)\}$, so $d((-1,0), z)=p+\max \{s \in p \mathbb{N}+$ 
$r \mathbb{N} \mid s \leq \rho(0,0)\}$. In the second case, $d((-1,0), z)=$ $d((0,0), z)+r-q=\rho(0,0)+r-q$. Using the notation $s_{(p r)}=\max \left\{s^{\prime} \in p \mathbb{N}+\operatorname{tr} \mathbb{N} \mid s^{\prime} \leq s\right\}$, we find

$$
\Delta \rho=\max \left(r-q, \rho(x)_{(p r)}-\rho(x)+p\right) .
$$

For all but a finite number of values for $\rho(x), \rho(x) \in$ $p \mathbb{N}+r \mathbb{N}$. In this situation, the point $z$ which maximizes $d((-1,0), z)$ in (4.4) lies on the segment $R S$, therefore $\rho(x)_{(p r)}=\rho(x)$ and $\Delta \rho=p$. Therefore, a table of the values of $\Delta \rho$ can be precomputed for those values of $\rho(x)$ which are not in $p \mathbb{N}+r \mathbb{N}$, while for other values $\Delta \rho=p$ can be used.

An expression similar to (4.6) can be found for the case where $y$ is an indirect neighbor of $x$. Note that for $p$ - $q$-r-metrics with small values of $p, q$ and $r$, we have $p+q-r=1$, such that $\Delta \rho$ will always be equal to $p$ or $p-1$.

These observations lead to the following algorithm.

Algorithm 4.5. Let $X$ be a bounded subset of $\mathbb{Z}^{2}$ and let $d$ be a $p-q-r$-metric.

(1) Compute the internal distance transform $\rho$ of $X$.

(2) For each $x \in X$ :

- If a direct neighbor $y$ of $x$ satisfies $\rho(y) \geq$ $\max \left(\rho(x)+r-q, \rho(x)_{(p r)}+p\right)$, mark $x$ as a non-medial axis point;

- If an indirect neighbor y of $x$ satisfies $\rho(y) \geq$ $\max \left(\rho(x)+r-p, \rho(x)_{(q r)}+q\right)$, mark $x$ as a non-medial axis point;

- If a knights move neighbor $y$ of $x$ satisfies $\rho(y) \geq \rho(x)+r$, mark $x$ as a non-medial axis point;

- otherwise mark $x$ as a medial axis point.

\section{The Opening Transform and the Pattern Spectrum}

In this section, we present the opening transform and the pattern spectrum, and an efficient algorithm for their computation, based on the medial axis. An operator $\alpha$ mapping subsets of a set $E$ to subsets of $E$ is called an opening [16] if it satisfies the following properties for each $X, Y \subseteq E$ :

(1) $X \subseteq Y \rightarrow \alpha(X) \subseteq \alpha(Y)$.

(2) $\alpha(\alpha(X))=\alpha(X)$.

(3) $\alpha(X) \subseteq X$.
From now on, we will restrict to the openings on the square grid $\mathbb{Z}^{2}$ and we will assume that this grid is provided with a chamfer metric. The most common opening is the structural opening, which can be constructed by "filling" a set with translates of a structuring elements: $X \circ A=\cup\left\{A_{h} \mid h \in E, A_{h} \subseteq X\right\}$. The structural opening $X \circ \bar{B}(r)$ of a set $X$ with a sphere of radius $r \in D$ is

$X \circ \bar{B}(r)=\cup\left\{\bar{B}(x, r) \mid x \in \mathbb{Z}^{2}, \bar{B}(x, r) \subseteq X\right\}$.

A granulometry or size distribution $[9,10]$ is defined as a family $\left\{\alpha_{r}\right\}$ of openings, where the range of $r$ is some ordered set and

$$
\alpha_{r} \alpha_{s}(X)=\alpha_{\max (r, s)}(X) .
$$

In this paper, the range of $r$ will be $D$. The family of structural openings with discs of a given radius is not a size distribution, but it is possible to construct a size distribution $\left\{\alpha_{r}\right\}_{r \in D}$ from them by

$$
\alpha_{r}(X)=\bigcup_{s \geq r} X \circ \bar{B}(s) .
$$

From the definition of a size distribution, it follows that $x \in \alpha_{r}(X) \Rightarrow x \in \alpha_{s}(X)$ for all $s \leq r$. This observation is the inspiration for the definition of the opening transform.

Definition 5.1. Let $X$ be a bounded subset of $\mathbb{Z}^{2}$ and let $\left\{\alpha_{r}\right\}$ be a size distribution. The opening transform $A_{X}$ is the mapping from $X$ to $D$ defined by

$$
A_{X}(x)=\max \left\{r \in D \mid x \in \alpha_{r}(X)\right\} .
$$

Important information on the shape of $X$ can be obtained by monitoring the change of $\alpha_{r}(X)$ as the parameter $r$ is varied. Maragos [9] defines the pattern spectrum (for subsets of $\mathbb{R}^{2}$, provided with the Euclidean metric) as

$$
p_{X}(r)=\frac{d A(X \circ B(r))}{d r},
$$

where $A(X \circ B(r))$ is the area of $(X \circ B(r))$. For the discrete case, this produces the following

Definition 5.2. [9] Let $X$ be a bounded subset of $\mathbb{Z}^{2}$ and let $\alpha_{r}$ be the size distribution induced by a chamfer 

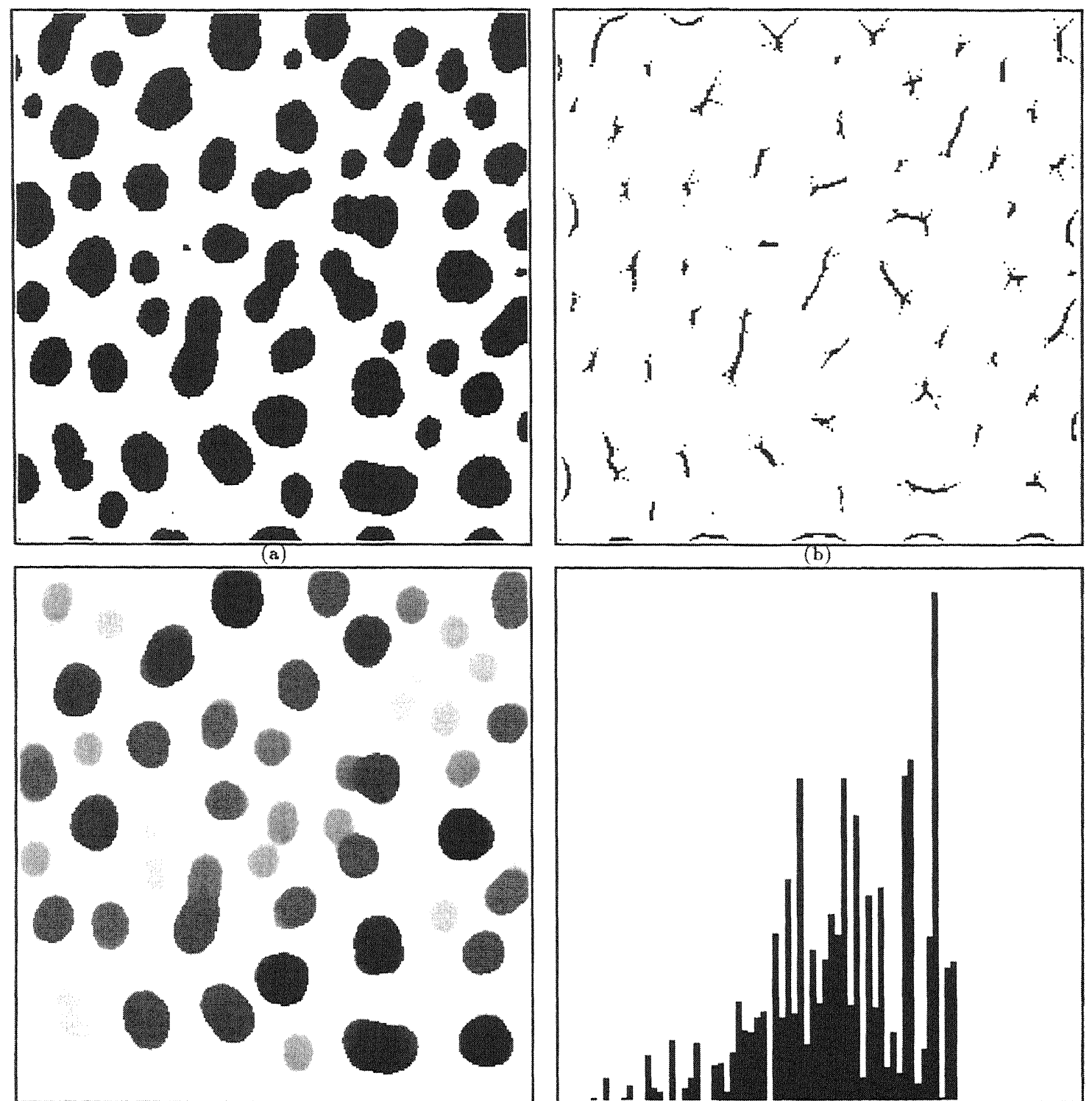

(c)

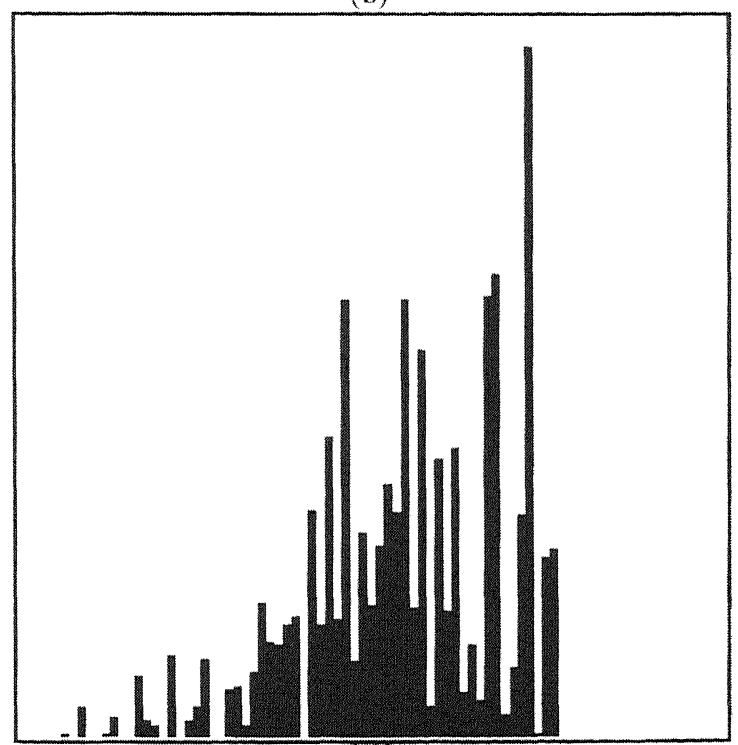

(d)

Figure 8. A binary image (a), its medial axis (b), its opening transform (c) and its pattern spectrum (d) as defined by the 5-7-11 metric. 
metric $d$. The the pattern spectrum $p(r)$ is the function from $D$ to $\mathbb{N}$ defined by

$$
p_{X}(r)=\left|\alpha_{r}(X)\right|-\left|\alpha_{r^{+}}(X)\right|,
$$

where $|\cdot|$ denotes the number of points in a set and $r^{+}=\min \left\{r^{\prime} \in D \mid r^{\prime}>r\right\}$ is the smallest element of $D$ which is larger than $r$.

Note that the pattern spectrum of a set is equal to the histogram of its opening transform. It is therefore possible to compute the pattern spectrum directly from the opening transform. In the rest of this section, we will therefore discuss only the opening transform.

From the definitions of $A_{X}$ and $\alpha_{r}$ it follows that

$$
\begin{aligned}
& A_{X}(x) \\
& =\max \left\{r \in D \mid x \in \bar{B}(y, r) \subseteq X \text { for some } y \in \mathbb{Z}^{2}\right\} .
\end{aligned}
$$

Because for each $x \in X$, the largest disc centered at $x$ and contained in $X$ has radius $\rho(x)$, this can be reduced to

$$
A_{X}(x)=\max \left\{\rho(y) \mid y \in \mathbb{Z}^{2}, x \in \bar{B}(y, \rho(y))\right\} .
$$

Observe that for each $x$ and $y$ such that $x \in \bar{B}(y, \rho(y))$, there is also a point $m$ in the medial axis $M_{X}$ such that $x \in \bar{B}(m, \rho(m))$. This leads to the relation

$$
A_{X}(x)=\max \left\{\rho(m) \mid m \in M_{X}, x \in \bar{B}(m, \rho(m))\right\} .
$$

This relation can be used to compute the opening transform by successively inspecting all maximal spheres in a bounded object $X$. First, all pixels in the result image are given value zero. The medial axis is computed, and each medial axis point is inspected in turn. If $m$ is the medial axis point being inspected, then all pixels in the sphere $\bar{B}(m, \rho(m))$ in the result image are visited. If the present value if the pixel in the result image is smaller than $\rho(m)$, the value is updated to $\rho(m)$. In the algorithm presented here, the medial axis points are sorted in order of increasing distance transform value and visited in this order. Thus, when the points in $\bar{B}(m, \rho(m))$ are being visited, a pixel in the result image can never have a value larger than $\rho(m)$, and the pixels in $\bar{B}(m, \rho(m))$ can always be assigned value $\rho(m)$, without prior inspection of the present pixel value. This strategy enhances the efficiency of the algorithm, because a comparison of pixel values in the inner loop is replaced by a sorting of the medial axis points, which must be performed just once. As $\rho(m)$ assumes only integer values smaller than some maximal value, the medial axis points can be sorted in linear time in the number of medial axis points using distribution sorting [8].

The efficiency of the algorithm depends on an efficient way of addressing all pixels in a sphere $\bar{B}(m, \rho(m))$. Certainly, computing such spheres in a two scan reconstruction algorithm would be too costly. In stead, the pixels in the sphere $\bar{B}(0, R)$, where $R$ is the largest occurring value of $\rho(m)$, are sorted in order of increasing distance to the origin. When the sorted list is computed, the pixels in each sphere $\bar{B}(0, r)$ for $r \leq R$ can be found by taking a suitable first part of the list. The pixels in spheres with different centers can be found by translation. In practice, the pixels in $\bar{B}(0, R)$ are not sorted, but computed in the correct order, and pixel positions are represented as relative offsets in the image array in order to provide fast access to the image.

Summarizing, the opening transform can be computed by the following:

Algorithm 5.3. The computation of the opening transform of a bounded subset $X$ of $\mathbb{Z}^{2}$ according to some chamfer metric $d$.

(1) Initialize the result image to 0 .

(2) Compute the distance transforms $\rho_{X}^{\mathrm{ext}}$ and $\rho$ and the medial axis $M_{X}$.

(3) Sort the medial axis points in order of increasing distance transform value.

(4) For all medial axis points, in increasing order:

- Set the pixels in $\bar{B}(m, \rho(m))$ to $\rho(m)$.

As noted before, the size distribution of $X$ is the histogram of $A_{X}$, so it can be computed from $A_{X}$ in a very straightforward way.

Figure 8 shows a binary image, its medial axis and its distance transform. Dark pixels correspond to large distance transform values. The computation of the opening transform took $0.43 \mathrm{~s}$, which is two orders of magnitude faster than the $45 \mathrm{~s}$ required by the brute force algorithm described in [13]. A similar reduction of computation time is found for other images.

\section{Conclusions}

In this paper, we presented a method for computing the medial axis defined by spheres in the $p-q-r$-chamfer 
metric. This result is an extension of the results in a previous paper [13], where an algorithm for the class of extending metrics was presented. The method is based on the understanding of the structure of shortest paths in the $p-q-r$-metric and of the structure of its range.

The $p-q-r$-metric approximates the Euclidean distance with an accuracy of $1.79 \%$, which is sufficient in most practical cases. The method proposed here produces a close approximation to the Euclidean medial axis. Yet the algorithm is very efficient, requiring only four image scans, and local computation in each scan.

Based on the medial axis transform, we presented an algorithm for the computation of the opening transform and the pattern spectrum associated with the 57-11 metric. The algorithm also uses a smart addressing scheme for pixels within a sphere of given center and radius. The approach based on the medial axis is two orders of magnitude faster than a brute force algorithm.

\section{Acknowledgments}

The author thanks H. Heijmans, F. Groen, and A. Toet for their discussions on contents and form of the manuscript. The author also thanks M. Schmitt, who suggested considering the extension of chamfer metrics to the continuous plane.

\section{References}

1. C. Arcelli and M. Frucci, "Reversible skeletonisation by $(5,7,11)$-erosion," in Visual Form-Analysis and Recognition, C. Arcelli, L.P. Cordella, and G. Saniti di Baja (Eds.), pp. 21-28, Plenum Press: New York, 1992.

2. C. Arcelli and G. Sanniti di Baja, "Finding local maxima in a pseudo-Euclidean distance transform," Computer Vision, Graphics and Image Processing, Vol. 43, pp. 361-367, 1988.

3. G. Borgefors, "Distance transformations in arbitrary dimensions," Computer Vision, Graphics and Image Processing, Vol. 27, pp. 321-345, 1984.

4. G. Borgefors, "Centres of maximal discs in the 5-7-11 distance transform," in Proceedings of the Eighth Scandinavian Conference on Image Analysis, 1993, pp. 105-111.

5. L. Dorst, "Pseudo-Euclidean skeletons," in Proceedings of the Eighth International Conference on Pattern Recognition, 1989, pp. 286-288.
6. H.J.A.M. Heijmans, Morphological Image Operators, Academic Press: Cambridge, Mass., 1993.

7. H.J.A.M. Heijmans and C. Ronse, "The algebraic basis of mathematical morphology part 1: Dilations and erosions, Computer Vision, Graphics and Image Processing, Vol. 50, pp. 245-295, 1990.

8. D. Knuth, The Art of Computer Programming Volume 3: Sorting and Searching, Addison-Wesley: Reading, Mass., U.S.A., 1973.

9. P. Maragos, "Pattern spectrum and multiscale shape representation," IEEE Transactions on Pattern Analysis and Machine Intelligence, Vol. 11, pp. 701-716, 1989.

10. G. Matheron, Random Sets and Integral Geometry, Wiley: New York, 1975.

11. G. Matheron, "Examples of topological properties of skeletons," in Images Analysis and Mathematical Morphology Volume 2: Theoretical Advances, J. Serra (Ed.), pp. 217-238, Academic Press: London, 1988.

12. F. Meyer, "Skeletons and perceptual graphs," Signal Processing, Vol. 16, pp. 335-363, 1989.

13. P.F.M. Nacken, "Chamfer metrics in mathematical morphology," Journal of Mathematical Imaging and Vision, Vol. 4, pp. 233253, 1994.

14. C.W. Niblack, D.W. Capson, and P.B. Gibbobs, "Generating skeletons and centerlines from the medial axis transform," in Proceedings of the Tenth International Conference on Pattern Recognition, 1990, pp. 881-885.

15. C. Ronse and H.J.A.M. Heijmans, "The algebraic basis of mathematical morphology part 2: Openings and closings, CVGIP: Image Understanding, Vol. 54, pp. 74-97, 1991.

16. J. Serra, Image Analysis and Mathematical Morphology, Academic Press: London, 1982.

17. E. Thiel and A. Montanvert, "Chamfer masks: Discrete distance functions, geometrical properties and optimization," in Proceedings of the 11th. International Conference on Pattern Recognition, 1992, pp. 244-247.

18. B. Verwer, "Local distances for distance transformations in two and three dimensions," Pattern Recognition Letters, Vol. 12, pp. $671-682,1991$.

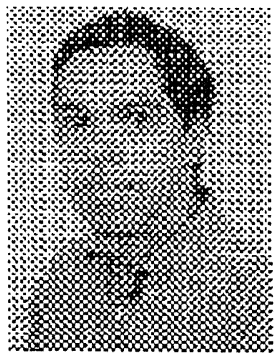

Peter Nacken (1966) has a Ph.D. in Image Analysis from the Univer sity of Amsterdam in The Netherlands. His research interests include mathematical morphology, hierarchical image processing algorithms and scale space, and three-dimensional image processing. Currently, he is working for Shell Research B.V. Rijswijk, The Netherlands. 